\title{
How Many Volunteers Does It Take To Map An Area Well? The validity of Linus' law to Volunteered Geographic Information
}

\author{
Mordechai (Muki) Haklay, Sofia Basiouka, Vyron Antoniou, Aamer Ather \\ Department of Civil, Environmental and Geomatic Engineering, \\ UCL, Gower St., London, WC1E 6BT \\ Tel. +44 2076792745 \\ m.haklay@ucl.ac.uk
}

KEYWORDS: Volunteered Geographical Information, OpenStreetMap, Linus’ Law, Spatial Data
Quality, Positional Accuracy

\begin{abstract}
In the area of Volunteered Geographical Information (VGI), the issue of spatial data quality is a clear challenge. The data that is contributed to VGI projects does not comply with standard spatial data quality assurance procedures, and the contributors operate without central coordination and strict data collection frameworks. However, similar to the area of open source software development, it is suggested that the data holds an intrinsic quality assurance measure through the analysis of the number of contributors who have worked on a given spatial unit. The assumption that as the number of contributors increases so does the quality is known as 'Linus' Law' within the Open Source community. This paper describes three studies that were carried out to evaluate this hypothesis for VGI using the OpenStreetMap dataset, showing that this rule indeed applies in the case of positional accuracy.
\end{abstract}

\section{Introduction}

Of the range of geographical information technologies that have emerged in the era of Web Mapping 2.0 (Haklay, Singleton and Parker, 2008; Elwood, 2009), the applications that involve geographical information collection and sharing by a wide range of participants deserve special attention, as they present a significant departure from past practices. Goodchild (2007) coined the term 'Volunteered Geographic Information' or VGI for this phenomenon.

The range of systems and applications that rely on VGI is very wide - from Google Map Maker, in which contributors add to the Google base mapping dataset, to scientific applications in which participants link accelerometers that are built into laptop computers to create a seismic monitoring network (Cochran et al., 2009). Within this range we can identify a class of systems that are truly based on volunteered information in the common sense of the word. These systems allow people from across the globe to create and share geographical content in an egalitarian manner, in what Benkler and Nissenbaum (2006) entitle 'Commons-Based Peer Production'. OpenStreetMap (OSM), which started at UCL in 2004, is a chief example of such a system. In OSM, volunteers (currently over 300,000 are registered to the site) contribute to create a free editable vector map of the world (see Haklay and Weber, 2008, for a detailed discussion). While many of the early volunteers of OSM were highly technically literate, they were not necessarily experts in geographical data collection. Data can be added to the project's database through digitising of location from aerial imagery, from tracks that were collected through a Global Positioning System (GPS) receiver or from notes that are taken during field surveys. As the project increases in size and provides new tools such as 'Walking Papers' (Migurski, 2009), which allows participants to print paper maps and use them for data collection activities, no assumption can be made about the background knowledge or data collection ability of volunteers.

In light of the data collection by amateurs, the distributed nature of the data collection and the loose coordination among them, how good is the quality of the information that is collected through such activities?' becomes a significant question. This is a crucial question about the efficacy of VGI activities and the value of the outputs for a range of applications, from basic navigation to more sophisticated 
applications such as site selection planning.

Research by Haklay (2010), Ather (2009), Kounadi (2009), Ueberschlag (2010), and Girres and Touya (in press), which focused on the evaluation of the road network and compared it to other sources of information, has demonstrated that, in terms of positional accuracy, the quality of OSM data is comparable to traditional geographical datasets that are maintained by national mapping agencies and commercial providers. Some of these studies (Haklay, 2010; Ather, 2009; Kounadi, 2009; Ueberschlag 2010) were based on the process that Goodchild and Hunter (1997) developed, which provides an estimation of the overlap between a reference dataset and a test dataset. In all these comparisons, the national mapping agency was used as the reference dataset and OSM as the test dataset. The results show overlap of about $80 \%$ in most cases but the values range from $100 \%$ down to $50 \%$ and below.

This range of values is not surprising, because the information is provided by many participants, who are acting independently and with loose coordination. However, this heterogeneity raises the need to understand at what stage of the data collection process the quality of the data becomes reliable, which is the topic of this paper.

One way of exploring the issue of ensuring quality in projects like OSM is to look for similar projects and see what types of quality assurance rules can be used. OSM, of course, is not the first commons-based peer-production activity. There are, in fact, many projects similar to it, albeit not in the area of geographical information. Examples are plenty - from Wikipedia, which focuses on sharing information (Wilkinson and Huberman 2007), to the Apache web server software. Many (although not all) open source projects have similar characteristics in terms of the distributed development effort and loose coordination amongst participants. Thus, parallels can be drawn between the quality issues of VGI and questions about code and software quality that were raised about many open source projects by the mainstream commercial software development community (Raymond, 2001).

While there are many mechanisms through which open source projects ensure the quality of the software (Halloran and Scherlis, 2002), the number of people engaged in it receive special attention in what is known as Linus' Law. A popular interpretation of the law states: 'Given enough eyeballs, all bugs are shallow' (Raymond, 2001, p.19). What this law means is that, in open source development projects, because multiple programmers are involved in the development and scrutinise the code, try it in different situations and improve it, the software code becomes increasingly better without formal quality assurance procedures. For mapping, this can be translated into the number of contributors that worked in a given area. The rationale behind it is that, if there is only one contributor in an area, he or she might inadvertently introduce some errors: for example, forgetting to survey a street or positioning a feature in the wrong location. However, several contributors might notice inaccuracies or 'bugs' and, therefore, the more contributors, the fewer 'bugs'. Spatial data quality includes many facets, such as positional accuracy, attribute accuracy or completeness. Therefore, there is a need to evaluate the validity of Linus' Law for the different aspects of spatial data quality.

If Linus' Law applies to VGI, it could provide an easy-to-calculate method for quality evaluation - for example, as in Haklay (2010), a regular grid can be used to count the number of contributors per square kilometre as a proxy for accuracy and can assist in decisions about fitness for use.

In the rest of the paper, we provide the details of a series of studies that were set to test if Linus' Law applies to one aspect of spatial data quality of VGI - positional accuracy - using the test case of OSM in England. We describe the methodology and results of three studies that were aimed at answering the core question: does Linus' Law apply to positional accuracy in VGI?

\section{Evaluating Linus' Law for OpenStreetMap}

The process of evaluating the validity of Linus' Law for VGI requires the following steps. First, there is a need to define what we mean by better quality. Next, this notion of better quality needs to be evaluated quantitatively for different areas, which also requires a clear definition. Once that is done, the number of contributors for each area can be counted and correlated to the measured 
quality.

There are multiple ways of evaluating spatial data quality, which are discussed briefly in Haklay (2010) and comprehensively in van Oort (2006). From the range of spatial data quality measures, we have selected positional accuracy as the first quality measure to be tested. There are several other quality measures such as attribute accuracy, completeness, etc. However, for this evaluation of Linus' Law, positional accuracy is a useful variable - it can be evaluated with a clear numerical output that represents the average positional accuracy of the areal unit under investigation, and it is also one of the aspects of VGI data quality that can be improved through the collaborative contribution of multiple contributors. The more contributors there are, the more likely it is that they will identify the road objects in their correct position. Thus the first step towards evaluating Linus' Law is the estimation of the positional accuracy of objects in a given area.

As hinted in the introduction, several quality tests have been carried out during recent years with OSM. The first one was carried out by Zulfiqar (2008) (discussed in Haklay 2010) comparing the OSM data to the Ordnance Survey Meridian 2 data so that the quality could be evaluated. This research revealed that the positional accuracy was quite high. Following this work, Ather (2009) studied four areas of London in detail, using the highly accurate MasterMap Integrated Transport Network (ITN) from Ordnance Survey (see Figure 1). Note that Meridian 2 is a generalised dataset, which while its nodes are kept in their original position, as was measured in high accuracy methods, the number of nodes in each road segment is reduced through the application of a 20-metre buffer. ITN, on the other hand, records the centreline of roads across the UK, based on information from field survey and through photogrammetry using high-resolution imagery. The accuracy of ITN is expected to be less than 1 metre in urban areas, where the study took place.

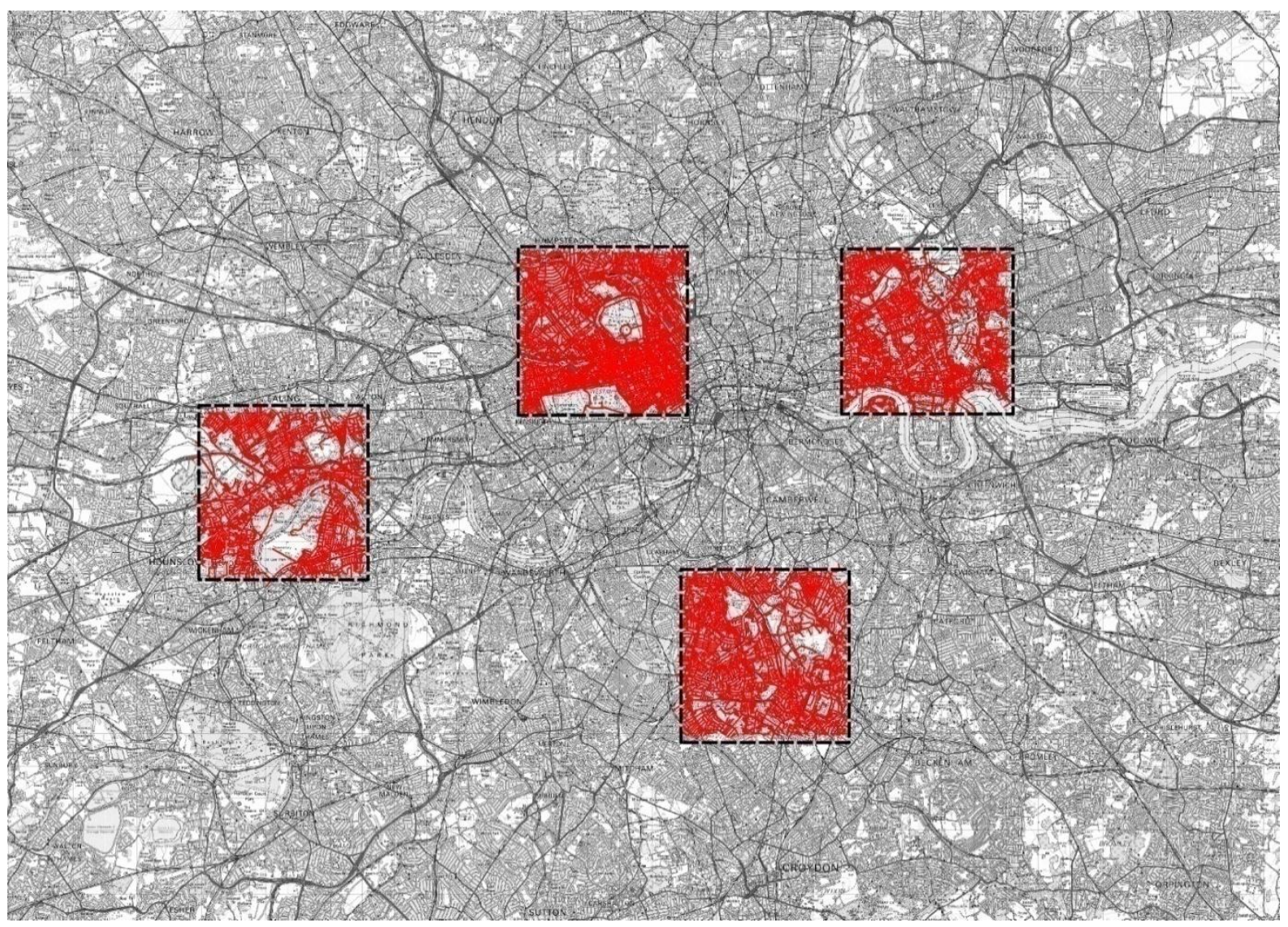


Figure 1: ITN data used for the comparison across London

Overall, the study evaluated 109 different roads in these four areas covering $328 \mathrm{~km}$, focusing on motorways, A-roads and B-roads. The area of interest comprises four tiles distributed in the Greater London area with two of the tiles being close to the city centre and the other two further out, in more suburban areas. Each tile covers a $25-\mathrm{km}^{2}$ area and was randomly chosen from the tiles that are available for London. London was chosen because OSM started in this city and, due to its magnitude, has attracted volunteers' interest while at the same time exhibiting a decrease in contribution towards the outer area of London, so the hypothesis can be tested.

As noted, the algorithm applied for the evaluation of positional accuracy is based on the 'simple positional accuracy measure for linear features' offered by Goodchild and Hunter (1997) and developed originally by Zulfiqar (2008) in Pitney Bowes Business Insight's MapBasic environment. The technique relies on a comparison of two datasets where the first is considered as the reference source, which is assumed to be of a high quality, and the second as the tested source. By applying the algorithm, a buffer of a specified width is created around the linear features of the reference source depending on the road category. A percentage of overlap is calculated for the total length of the tested data that lies within the buffer around the referenced one. The data provided by Ordnance Survey was considered as the 'referenced' and the OSM data as the 'tested'. The following buffer widths were used, corresponding to the real width of the roads using estimations obtained from the House of Commons Hansard Written Answers (Hansard 2003).

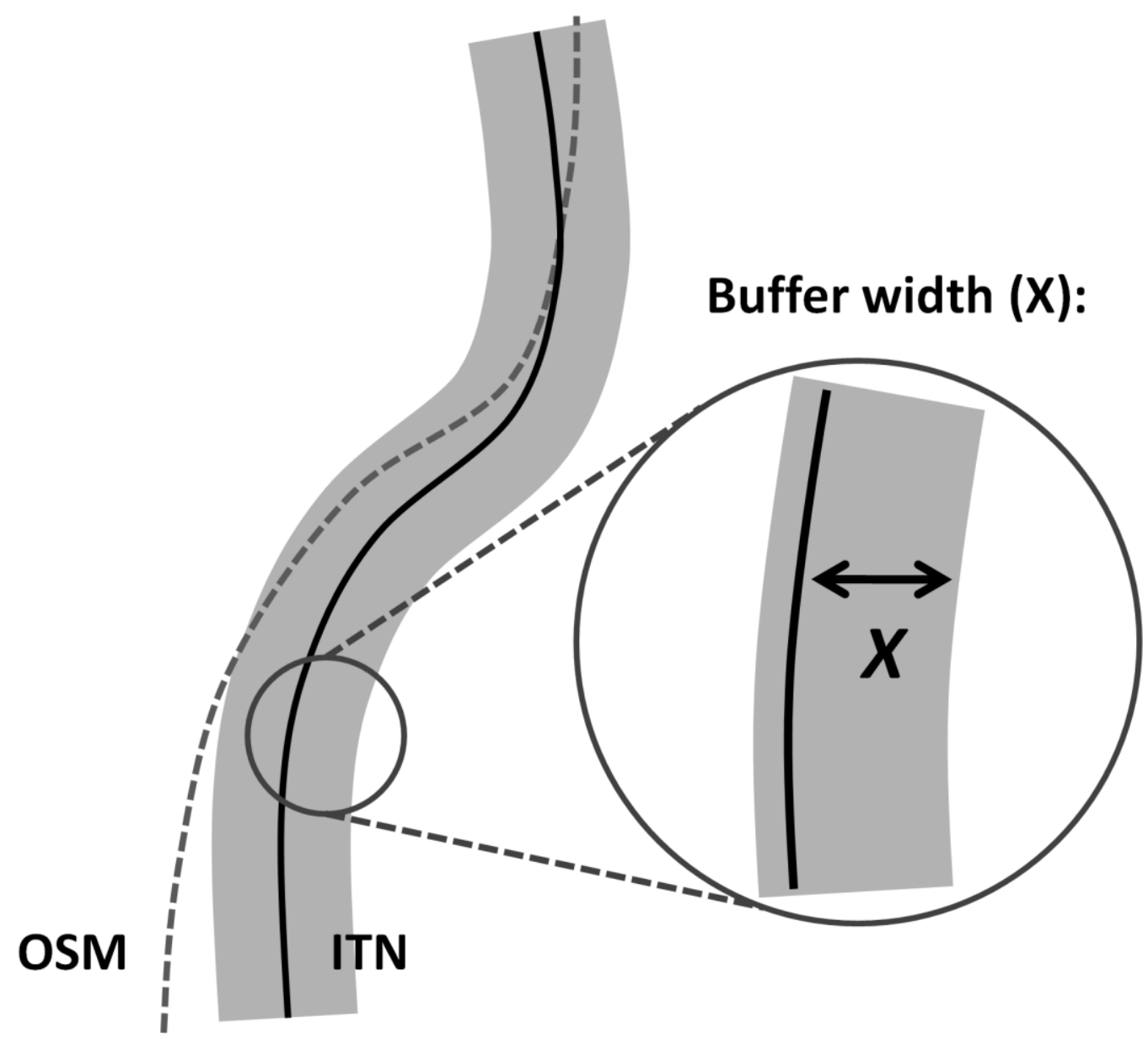

Figure 2: Example of the buffer-zone method. A buffer of width $\mathrm{x}$ is created around a high- 
quality object, and the percentage of the tested object that falls within the buffer is evaluated

For motorways, an 8-metre buffer zone was applied. For A-roads, a 5.6-metre buffer zone was created and for B-roads, a 3.75-metre buffer zone.

An editing process was necessary so that, by labelling the features correctly, the road name attribution matched between the two datasets. The buffer analysis was applied in each tile and for each road category separately. After applying the buffer analysis for each road category separately, the average positional accuracy was calculated for all records. The results indicated that the percentage of the positional accuracy of OSM is high and the overlap exceeded $80 \%$. The percentage reached $85.80 \%$ in South London, $85.19 \%$ in the North, $81.03 \%$ in the West and $80.80 \%$ in East London. It is noticeable that the percentage of the positional accuracy in one in four roads was between $95 \%$ and $100 \%$ and in more than half of the roads was over $85 \%$.

Taking into account road categories, it was found that the primary roads were mapped better, offering higher percentages of positional accuracy, although it is important to remember that, because of the road width, there is a greater likelihood that OSM features will fall within the buffer. The motorway achieved the highest percentage followed by the A-roads and finally by the B-roads. It is important to remember that it is easier to locate a line that falls inside a motorway, which is a six-lane object, than in a narrow street, so the differences in overlap and not surprising.

The contributor analysis was based on user name details that were downloaded from the OSM website as an XML file. The analysis was carried out in two stages. First, the number of contributors per area was compared to the average positional accuracy of the tile, which is based on the value of all the roads that pass through it. The second stage consisted of a more detailed approach of the analysis by dividing each tile into 1-square-kilometre grids. Across the four test regions, 100 grid squares were analysed. The contributors in each grid were counted in comparison to the roads. Cycle routes, steps and pathways were excluded from the linear features so that only the roads that were used in the previous analysis were evaluated in the contributor analysis.

\begin{tabular}{|l|r|r|r|}
\hline & $\begin{array}{r}\text { Average } \\
\text { accuracy }\end{array}$ & $\begin{array}{r}\text { Number of } \\
\text { contributors }\end{array}$ & $\begin{array}{l}\text { Average length of } \\
\text { road per contributor }\end{array}$ \\
\hline $\begin{array}{l}\text { TQ28se } \\
\text { (North/Central) }\end{array}$ & $85.19 \%$ & 145 & 1.768 \\
\hline TQ38se (East) & $80.80 \%$ & 91 & 1.203 \\
\hline TQ37sw (South) & $85.80 \%$ & 56 & 1.204 \\
\hline TQ17ne (West) & $81.03 \%$ & 81 & 1.017 \\
\hline
\end{tabular}

Table 1: Overall quality statistics for the four study areas

On a general level, the evaluation showed that all the test regions chosen were covered by a large number of contributors. Moreover, the results of the first contributor analysis showed that the North London tile had by far the largest number of contributors (145) and also the greatest ratio of total length of road to number of contributors (1.768). The tiles having the lowest percentages of positional accuracy (East and West) also had the lowest ratio. The only exception was the South London tile, which didn't follow the general trend. The interesting result here is 
that, although the average length per contributor increases, the average accuracy increases, too. However, this level of spatial analysis, over 25 square kilometres, is too coarse to provide robust analysis of the number of contributors and the impact of positional accuracy.

The second part of the analysis showed that most of the grid squares were edited by between 5 and 20 contributors and none of them by fewer than 5 contributors. This is in contrast with the general situation in 2008, where Haklay (2010) demonstrated that $89.5 \%$ of England was covered by 3 or fewer contributors.

Comparing the number of contributors to road name completeness showed a slight positive trend (Figure 3), though not statistically significant. However, because the tests use the number of contributors for each square kilometre while the positional accuracy is calculated for the full length of the road, the results are inconclusive. More importantly, once the research was carried out, it became apparent that VGI cannot be evaluated at the object level when it comes to long roads that span several kilometres as different parts of the roads might be contributed to and corrected by different individuals. At the same time, the analysis needed to focus on areal properties and not on individual contributor ability to digitise information correctly. Therefore, breaking the area into smaller units and evaluating the number of contributors with the average quality in each area seemed a promising direction in which to continue this investigation.

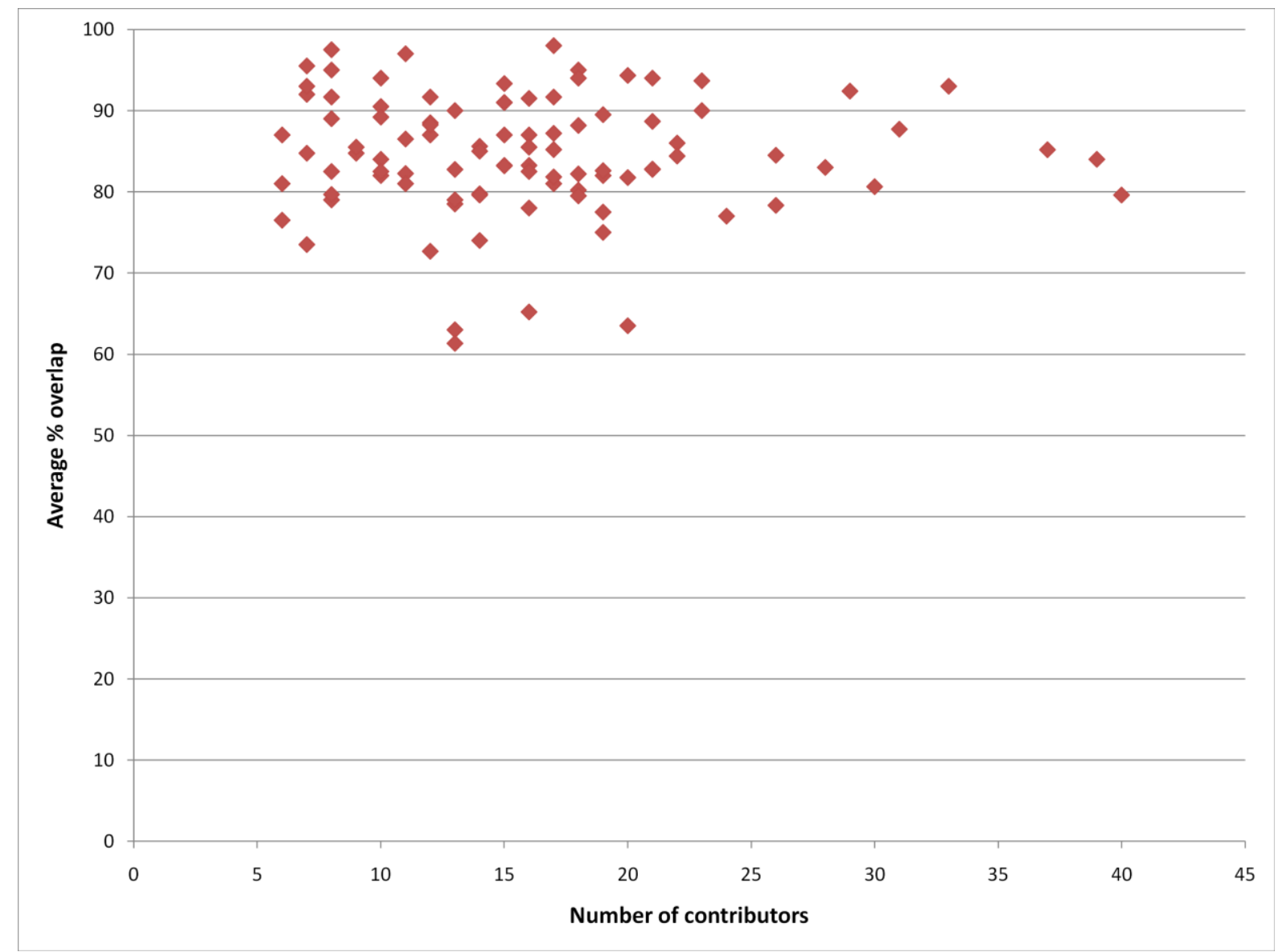

Figure 3: Number of contributors and estimated positional accuracy - calculated at object level

This new direction was taken by Basiouka (2009). The analysis focused on the same areas in a different manner.

The positional accuracy was evaluated through a detailed process after having divided each of the areas into 25 separate square kilometre cells. The roads were segmented at the edges of each 
grid to allow the evaluation of local positional accuracy. The algorithm was modified so that the buffer analysis was performed in each grid and each road category separately. The average positional accuracy of each grid was calculated as the weighted mean of the length of the roads and the positional accuracy of every road.

The evaluation shows that positional accuracy at the tile level is high across the study area. The percentages of overlap between the two datasets fluctuated from $83.7 \%$ for the West London area to $89.3 \%$ for the South London area. The East London area had an average percentage of positional accuracy approaching $84.6 \%$ and the North London area had 86.6\%. The minor differences between these results and Table 1 are due to the calculation of the average percentages of the positional accuracy.

With this new analysis, the number of contributors was correlated to the average percentage of positional accuracy. Ranking of the grids according to the number of contributors to the mapping showed that $29 \%$ (almost one third) of the grids was mapped by 16 to 20 contributors and $27 \%$ of grids by 11 to 15 contributors.

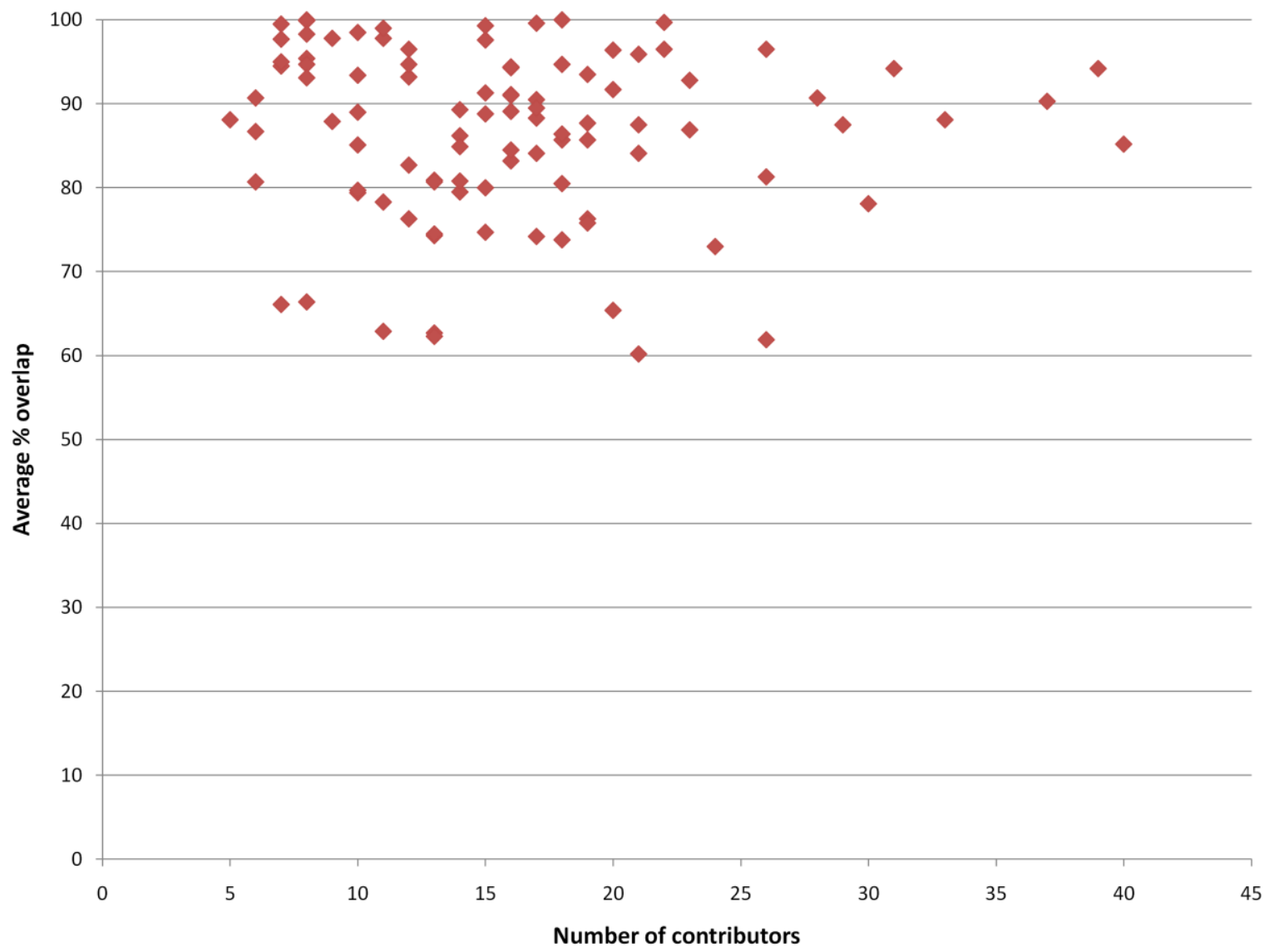

Figure 4: Number of contributors and estimated positional accuracy - calculated for each grid square

The results (Figure 4) show that, above 5 contributors, there is no clear pattern of improved quality as the number of contributors increases and the results are not statistically significant. The graph shows that the quality, while generally very high, is not dependent on the number of contributors beyond a certain number - so Linus' Law does not apply to OSM in a simplistic way (and possibly not to VGI in general). That said, having 5 contributors or more is likely to lead to quality that is high (notice that, in Figure 4, over $93 \%$ of the data points are above $70 \%$ ).

In an effort to move the evaluation of the positional accuracy quality element of the OSM road network from test sites in urban areas to a national level, Antoniou (forthcoming) developed a different 
methodology that used the geometric position of the Ordnance Survey (OS) Meridian 2 road intersections to evaluate positional accuracy. Although, as noted, Meridian 2 is constructed by applying a 20-metre generalisation filter to the centrelines of the OS Roads Database, this generalisation process does not affect the positional accuracy of node points and thus their accuracy is the best available (OS, 2009). Goodchild and Hunter (1997) note that the use of the road intersections can be used to examine the positional accuracy of road network data against a reference dataset. They do raise a word of caution though regarding the need to ensure a good match of corresponding nodes in both datasets. Therefore an algorithm was developed for the identification of the correct nodes between the two datasets, and the average positional error was calculated for each square kilometre of the National Grid for England (Figure 5). Note that areas where no calculation was carried out are left empty on the map.

The algorithm, which is repeated for each Meridian 2 node, starts by searching for the OSM node that is the nearest to a given node from the Meridian 2 dataset. This is carried out by searching for the minimum distance between the Meridian 2 node and the OSM nodes that fall under a threshold of 40m. The algorithm then verifies that the selected OSM node is not closer to another Meridian 2 node. If there is no other Meridian 2 node closer to the selected OSM node, this pair of nodes is recorded as a possible positive match. If the distance between the two nodes is equal or less than $15 \mathrm{~m}$ (which is approximately the positional accuracy of a hand-held GPS device), and also taking into consideration the uncertainty of the Meridian 2 accuracy according to the scale of origin, then the node match is directly assumed to be correct. Otherwise (i.e. the distance between the two nodes is $15 \mathrm{~m}-40 \mathrm{~m}$ ), a second level of verification is introduced. Here the verification takes place through the examination of the road names that intersect each of the paired nodes. The algorithm finds out and records the number of road segments that start (or end) from the specific node of each dataset and how many of those segments have a name. Then the harmonised names are compared and the number of matched names is recorded.

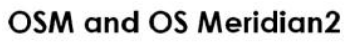

Average positional error $(\mathrm{m})$

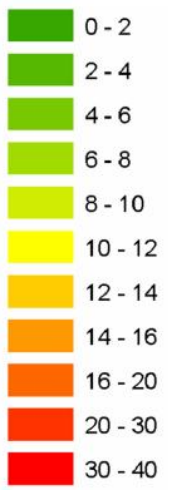

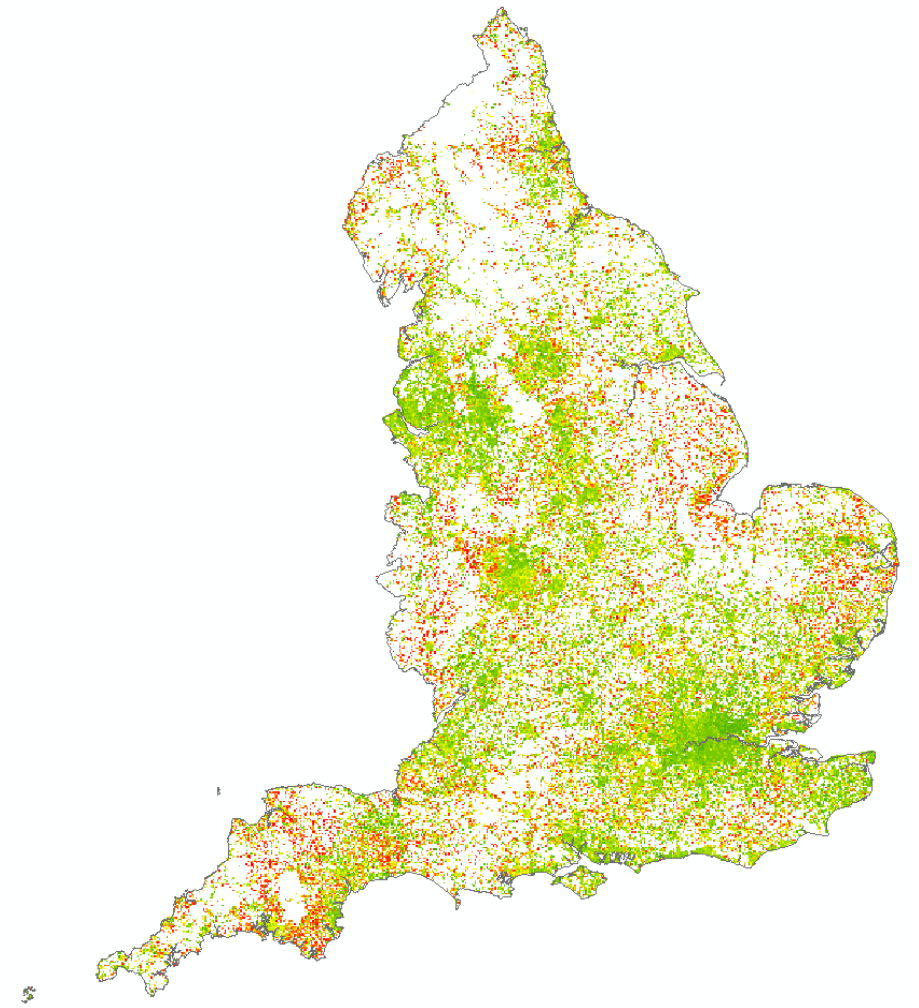

Figure 5: The evaluation of the OSM positional accuracy against the OS Meridian 2 intersections

It is evident that the most accurate tiles are located in major urban areas such as London, Liverpool, 
Manchester or Birmingham. In contrast, tiles in the rural areas have generally larger positional errors. Nevertheless, the overall positional accuracy is considerably high (Figure 6) given the means that contributors have at their disposal for the OSM data collection. More than $70 \%$ of the intersections have a positional error smaller than 12 metres and another 10\% between 12 and 15 metres.

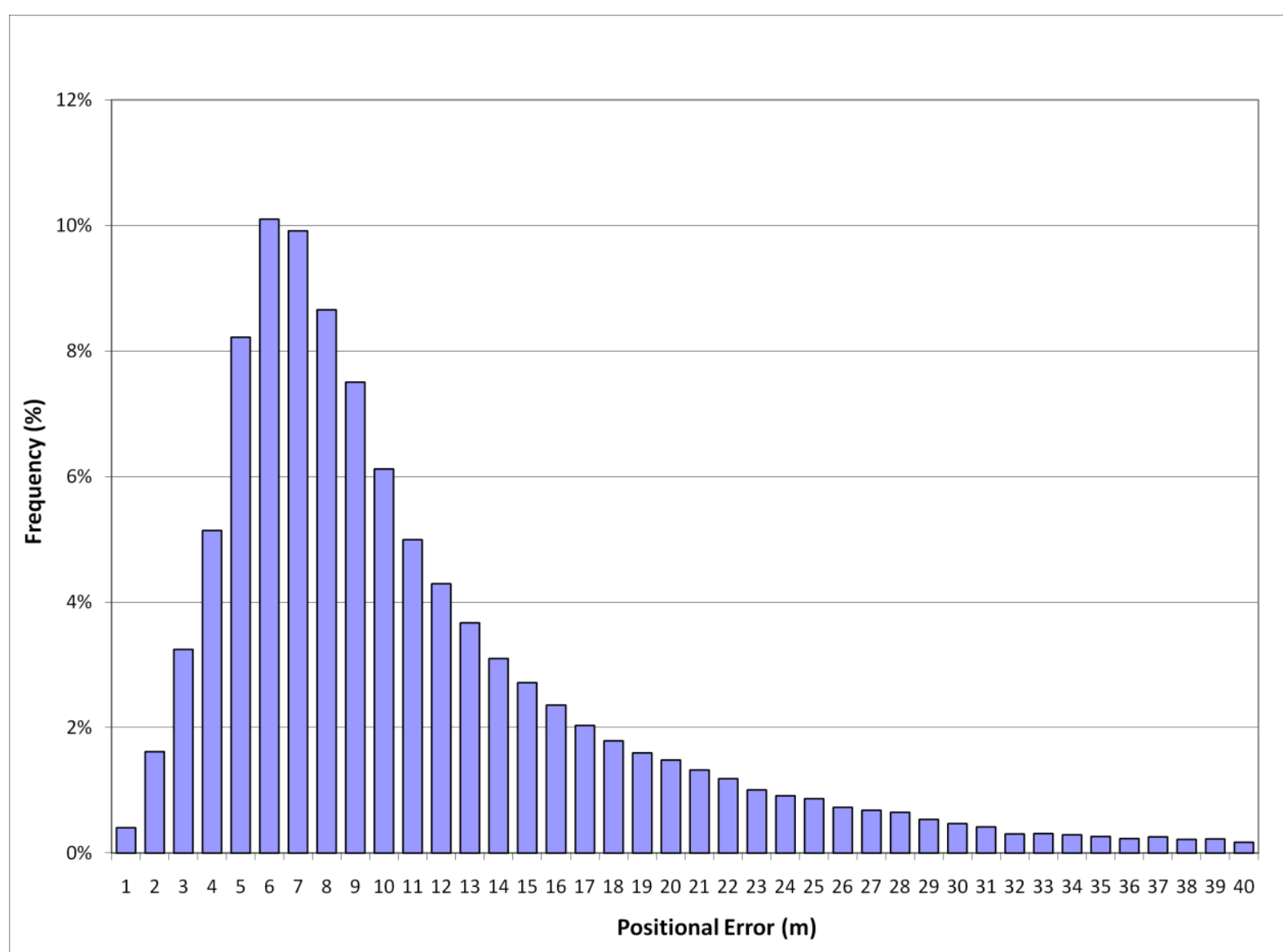

Figure 6: Frequencies of the positional errors of OSM data against the OS Meridian 2

The data collection regarding the OSM positional accuracy for England enabled two separate analyses at a national level (Figure 7) to be performed. The first analysis involved a comparison between contributors and average positional accuracy for all grid squares with the same number of contributors. Here, in contrast with the results yielded when examining the phenomenon in the four London test sites, it is clearly shown that the number of contributors to an area affects the positional accuracy of the OSM dataset. For the full dataset of over 43,000 square kilometre the correlation is weak (-0.143) but significant at the 0.01 level (2-tailed). For the average value each category of contributors, the correlation is strong (-0.844) and significant at the 0.01 level (2-talled). Yet, this statement is valid up to a certain extent. It can be seen that positional accuracy remains level when the number of contributors is approximately 13 or more. It is important to note that up to 13 there are over 20 grid squares in every category, and in the higher categories there is sometimes only a single cell. Until the number of contributors reaches approximately 13 , each one of the contributors considerably improves the dataset's quality. Consequently, Linus' Law affects the quality of OSM data and potentially other VGI sources as well when the number of contributors is small, but there is a decreased gain in terms of positional accuracy when the number of contributors passes 13. 
The Cartographic Journal Vol. 47 No. 4 pp. 315-322 November 2010

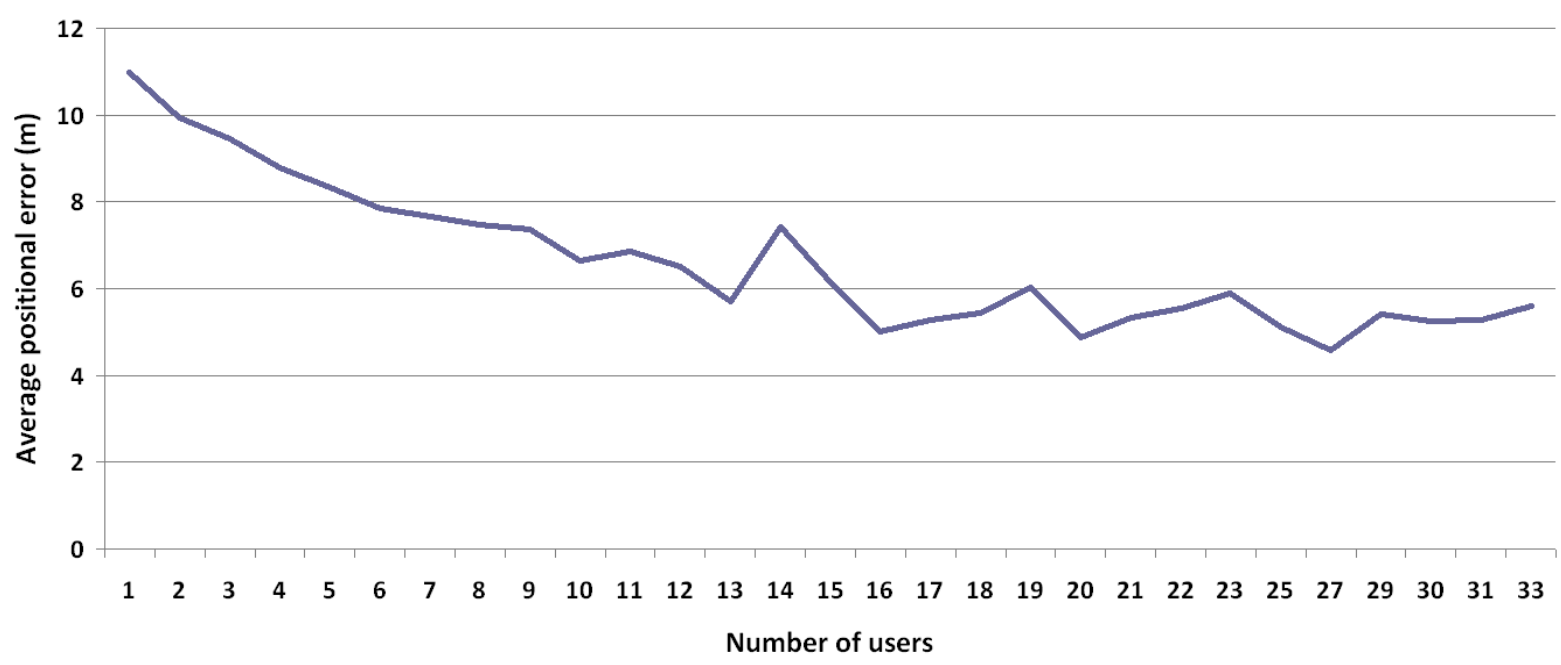

Figure 7: Average positional error vs. number of contributors for OSM road network for England

The second analysis is based on completeness data presented by Haklay (2010), which showed that the level of completeness is linked with socio-economic factors as OSM contributors provide less coverage to poor and marginalised areas compared to richer areas. Figure 8 shows the frequencies of the average positional accuracy for the 1- square kilometre cells where the OSM completeness level is higher (blue) and lower (purple) than the OS Meridian 2 dataset.

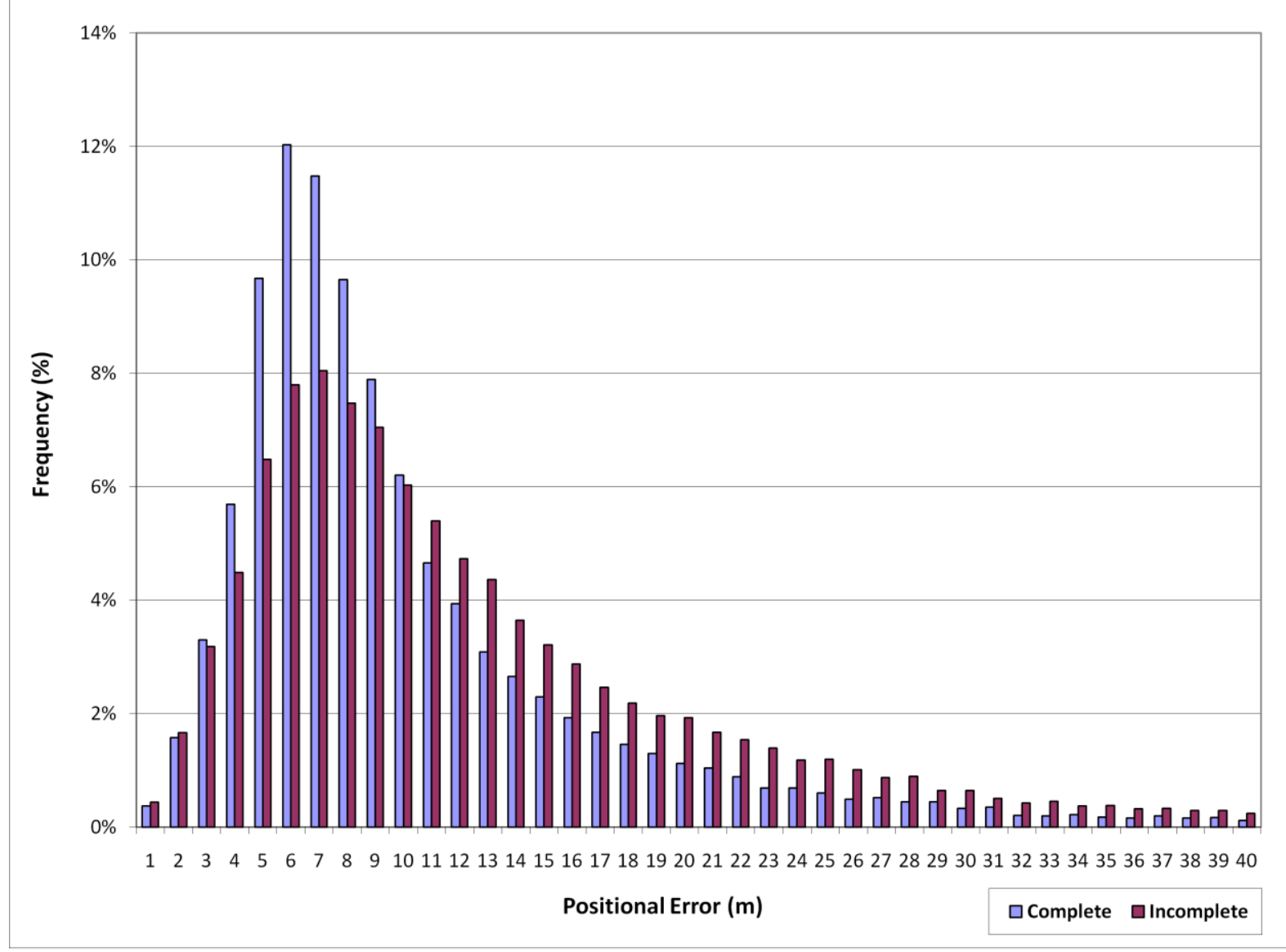

Figure 8: Average positional error vs. frequency for OSM road network for England 
Again it is evident that the positional accuracy in the complete areas is considerably better than the accuracy of the incomplete areas. Indeed, the average positional error for the former is $9.57 \mathrm{~m}$ with the standard deviation equal to $6.51 \mathrm{~m}$. The average positional error for the incomplete tiles is $11.72 \mathrm{~m}$ while they also have a greater standard deviation of $7.73 \mathrm{~m}$. This means that the OSM data for the incomplete areas is almost $22.5 \%$ less accurate.

This last experiment does not relate to the quantity of the 'eyeballs' mentioned in Linus' Law rather to their quality. It has been shown that, for an area to be completely and accurately covered, not only does it need more 'eyeballs' but also 'eyeballs' that are undeterred by an area's perceived reputation and that are willing to look through the curtain that socio-economic barriers draw.

\section{Conclusions}

In this paper, we have shown that, while Linus' Law applies to OSM and we speculate that it might be valid to VGI in general, the relationship between the number of contributors and the quality of the data is not linear. The conclusions of our studies are that, beyond 15 contributors per square kilometre, the positional accuracy becomes very good below 6 metres. At the other end of the scale, the first 5 contributors to an area seem to provide the biggest contribution in terms of positional accuracy improvement.

There are three other points that are important for VGI research and which emerge from our investigations.

First, the importance of heterogeneity. Our research demonstrates that VGI datasets must be approached as heterogeneous datasets that should be evaluated locally and not globally. We chose a spatial unit of 1 square kilometre for our studies, and, in our view, much larger units would start conflating the actions of multiple contributors, thus becoming less helpful in understanding quality issues. The size of areal unit that is suitable for VGI studies is an issue that deserves more research.

Second, this heterogeneity means that, for people who want to use VGI datasets, there is a significant challenge of understanding this aspect and evaluating it - as from a user perspective it is more difficult to grasp what the overall quality is for a given area. Thus, despite our recommendation that the areal unit must be small, it can be useful to provide aggregate values for large units that are of interest to the enduser - for example, a local authority boundary. How to do that in a way that is meaningful to the enduser while accurately conveying the variability of the quality across the area is another research challenge.

Third, we have demonstrated that it is possible to consider spatial data quality indicators that are intrinsic to the dataset itself, without the use of a reference dataset. Linus' Law is such an indicator. We would like to see other studies that use different methods to test our findings, such as the study by Girres and Touya (in press) which found that 'the number of OSM objects in an area clearly grows in relation to the number of contributors in the area, but in a non-linear way: most areas have less than one contributor per $\mathrm{km}^{2}$ and few contributions; the areas with one to three contributors per $\mathrm{km}^{2}$ have five to ten times more contributions; the areas with more than three contributors per $\mathrm{km}^{2}$ have ten to a hundred times more contributions which shows a kind of competitiveness between contributors when there are many'. Through these emerging studies it might be possible to develop 'bootstrapping' spatial data quality indicators that help in decisions about the fitness for use of VGI datasets.

There are open questions that deserve more research - for example, understanding the relationship not only between the number of contributors and data quality, but also to understand if there is a difference in quality following the amount of data that is contributed by each person. There is also a need to understand the impact of the number of contributors on attribute accuracy. Throughout the paper we have focused on positional accuracy as the measure of spatial data quality, although we have just touched upon the issue of completeness. It is, therefore, necessary to continue this evaluation on other aspects of quality including attributes and up-to-dateness of VGI data sources as well as evaluations that look at other datasets. 


\section{Acknowledgements}

The Ordnance Survey External Research and University Liaison who provided the Meridian 2 dataset for this study in January 2008. The 1:10,000 raster and the SOA boundaries were provided under EDINA/Digimap and EDINA/UKBorders agreements.

All maps are Ordnance Survey (C) Crown copyright. Crown copyright/database right 2008, Ordnance Survey/EDINA supplied service, and Crown copyright/database right 2008. OSM data provided under Creative Commons and attributed to OpenStreetMap. All rights reserved.

\section{References}

Antoniou, V. (forthcoming) User Generated Spatial Content: An Analysis of the Phenomenon and its Challenges for Mapping Agencies. PhD thesis, Civil, Environmental and Geomatic Engineering, UCL

Ather, A. (2009) 'A Quality Analysis of OpenStreetMap Data' unpublished M.Eng. dissertation, UCL

Basiouka, S., 2009. Evaluation of the OpenStreetMap quality, unpublished MSc dissertation, London, University College London.

Benkler, Y. and Nissenbaum, H. (2006) Commons-Based Peer Production and Virtue. Journal of Political Philosophy, 14 (4): 394-419

Cochran, E.S., Lawrence, J.F., Christensen, C. and Jakka, R.S. (2009) The Quake-Catcher Network: Citizen Science Expanding Seismic Horizons, Seismological Research Letters (January 2009), 80(1):26-30

Elwood, S. (2009) Geographic information science: new geovisualization technologies - emerging questions and linkages with GIScience research, Progress in Human Geography, 33:2, 256-263

Girres, J.F. and Touya, G. (2010) Quality assessment of the French OpenStreetMap dataset, Transactions in GIS, 14(4):435-459

Goodchild, M. F. and Hunter, G. J. (1997) A simple positional accuracy measure for linear features, International Journal of Geographical Information Science, 11(3): 299-306

Goodchild, M.F. (2007) Citizens as Voluntary Sensors: Spatial Data Infrastructure in the World of Web 2.0, International Journal of Spatial Data Infrastructures Research, 2: 24-32

Haklay, M. (2010) How good is OpenStreetMap information? A comparative study of OpenStreetMap and Ordnance Survey datasets for London and the rest of England, Environment and Planning B, 37: 682703

Haklay, M.. and Weber, P. (2008) OpenStreetMap - User Generated Street Map, IEEE Pervasive Computing. October-December 2008, 12-18

Haklay, M., Singleton, A. and Parker, C. (2008) Web Mapping 2.0: the Neogeography of the Geoweb, Geography Compass 3: 2011-2039, doi: 10.1111/j.1749-8198.2008.00167.x

Hansard (2003) HC Vol. 412 Col. 340W (30 Oct 2003)

Halloran, T.J. and Scherlis, W.L. (2002) High quality and open source software practices. Meeting Challenges and Surviving Success: 2nd Workshop on Open Source Software Engineering, University College Cork, Ireland, 2002. Available at: http://opensource.ucc.ie/icse2002

Kounadi, O. (2009) 'Assessing the quality of OpenStreetMap data', unpublished MSc dissertation, UCL

Migurski, M. (2009) Walking Papers (walking-papers.org)

Ordnance Survey (2009) Meridian 2. User Guide and Technical Specification. Available at:

http://www.ordnancesurvey.co.uk/products/meridian2/pdf/meridian2userguide.pdf

Raymond, E.S. (2001) The Cathedral and the Bazaar, O’Reilly, p.19

Ueberschlag, A. (2010) A first assessment of the OpenStreetMap quality in Switzerland, Unpublished manuscript EPFL, Switzerland 
Van Oort P.A. J (2006) Spatial Data Quality: From Description to Application, PhD thesis, Wageningen University

Wessa P. (2008) Pearson Correlation (v1.0.3) in Free Statistics Software (v1.1.23-r6), Office for Research Development and Education, URL http://www.wessa.net/rwasp_correlation.wasp/

Wilkinson, D. M. and Huberman, B. A. (2007) Cooperation and quality in wikipedia. In WikiSym '07: Proceedings of the 2007 international symposium on Wikis, pages 157-164, New York, NY, USA, ACM

Zulfiqar, N. (2008) A Study of the Quality of OpenStreetMap.org maps: A comparison of OSM data and Ordnance Survey Data, MEng Thesis, London, University College London

\section{Biography}

Mordechai (Muki) Haklay is a senior lecturer in Geographical Information Science in the department of Civil, Environmental and Geomatic Engineering at UCL. His research interests are in public access to environmental information, Human-Computer Interaction (HCI) and Usability Engineering for GIS, and societal aspects of GIS use.

Aamer Ather holds an M.Eng. in Geoinformatics from University College London. For his final year thesis he investigated the quality and accuracy of OpenStreetMap data using GIS software. After graduating in 2009, he is now exploring various career opportunities and is currently in employment with Infotech, working on a project with the Rural Payments Agency.

Sofia Basiouka is a PhD student in the department of Rural and Survey Engineering at the National Technical University of Athens (N.T.U.A). She recently completed the MSc in GIS at UCL (2009) and the M.Eng. in Survey Engineering at N.T.U.A.(2008). Her research is focused on Cadastre.

Major Vyron Antoniou serves as an officer in the Greek Army and, since 1998, he has been posted at the Hellenic Military Geographical Service. Currently he is a PhD student at UCL in the department of Civil, Environmental and Geomatic Engineering. His research interests are in user-generated spatial content and mapping agencies, spatial databases, vector data transmission over the $W e b$ and web mapping. 